\title{
Quelle intégration pour les réfugiés palestiniens du Liban? Etude à partir des mariages libano- palestiniens
}

The integration of Palestinian refugees in Lebanon: Lebanese-Palestinian marriages

Die Integration der palästinensischen Flüchtlinge im Libanon: libanesischpalästinensischen Ehen

\section{Daniel Meier}

\section{OpenEdition \\ Journals}

Édition électronique

URL : http://journals.openedition.org/rge/2021

DOI : 10.4000/rge.2021

ISSN : 2108-6478

Éditeur

Association des géographes de l'Est

Édition imprimée

Date de publication : 31 décembre 2009

ISSN : 0035-3213

\section{Référence électronique}

Daniel Meier, «Quelle intégration pour les réfugiés palestiniens du Liban ? Etude à partir des mariages libano-palestiniens », Revue Géographique de l'Est [En ligne], vol. 49 / 4 | 2009, mis en ligne le 01 novembre 2010, consulté le 08 septembre 2020. URL : http://journals.openedition.org/rge/2021 ; DOI : https://doi.org/10.4000/rge.2021

Ce document a été généré automatiquement le 8 septembre 2020

Tous droits réservés 


\title{
Quelle intégration pour les réfugiés palestiniens du Liban ? Etude à partir des mariages libano- palestiniens
}

\author{
The integration of Palestinian refugees in Lebanon: Lebanese-Palestinian \\ marriages \\ Die Integration der palästinensischen Flüchtlinge im Libanon: libanesisch- \\ palästinensischen Ehen
}

Daniel Meier

1 Beaucoup a été écrit sur les réfugiés palestiniens au Moyen-Orient ou sur les sociétés des différents pays de cette région. Par contre, comme me le disait Yezid Sayigh lors d'une rencontre à Paris, bien peu a été fait sur les relations entre Palestiniens et nationaux des pays où ils vivent en exil. Le cas du Liban s'imposait alors comme lieu d'étude au vu de la situation difficile que les Palestiniens y connaissent. Toutefois, un des défis consistait à trouver un angle d'attaque de la problématique des relations libano-palestiniennes qui ne soit ni sécuritaire ni exclusivement juridico-politique et qui puisse néanmoins montrer en acte un processus social.

2 Dans ces conditions, les relations matrimoniales entre ces deux groupes m'ont paru un objet à la fois original et nodal dans le cadre d'une analyse de sociologie politique visant à explorer leurs modes d'insertion sociale. De surcroît, le mariage peut être appréhendé comme une modalité d'intégration des Palestiniens au collectif national libanais, dans ses dimensions sociales et économiques ${ }^{1}$ alors même que le discours dominant est plutôt celui d'un pays qui ne veut pas devenir une terre d'accueil pour les Palestiniens. Dans les lignes qui suivent, je tenterais de démontrer que les unions matrimoniales entre ces deux groupes nationaux fonctionnent, dans le Liban d'après-guerre, comme un système de pratiques intégratif tout en reconduisant les formes d'exclusion déjà à l'œuvre dans les sphères économique et juridique. 
3 La centralité de la question identitaire me conduira, dans un premier temps, à interroger la place et les enjeux du mariage dans la société libanaise en regard du poids social et politique des communautés. Dans un second temps, j'examinerai les alliances matrimoniales libano-palestiniennes à partir d'un corpus d'entretiens facilitant l'appréhension de la problématique dans une perspective diachronique. Ces pratiques mettront à jour les différents types de groupes libano-palestiniens que l'après-guerre laisse voir ainsi que les dynamiques d'exclusion qui caractérisent leur intégration sociale.

\section{Le cadre institutionnel : Etat et mariage civil}

4 Les spécialistes du Liban s'accordent à souligner qu'un des acteurs phares de la scène politique libanaise est le groupe communautaire qui est en fait un groupe religieux au Liban, comme les chrétiens maronites ou les musulmans druzes. Ces groupes sont régulièrement impliqués dans des tractations politiques et semblent utiliser leurs membres comme des corps homogènes et unifiés autour de l'appartenance communautaire. Leurs pratiques et calculs politiques trouvent sa raison d'être dans la place dévolue aux communautés au sein du système politique libanais. Elles opèrent comme des instances de médiations capables d'intervenir entre le citoyen et l'Etat et ce depuis l'époque ottomane. Lors de la chute de ce dernier, le régime communautariste mis en place en 1864 " fut maintenu intégralement par l'Acte du mandat de la SDN qui instaura l'égalité en droit de tous les individus par le travers de l'égalité en droit de toutes les communautés » (Assaf, $2000: 25$ ). Dans l'article 9 de la Constitution, rédigée en 1926, l'État se déclare neutre à l'égard de toutes les communautés tout en reconnaissant la liberté de croyance à tous les citoyens. Mais, l'arrêté 60 (du 13.3.1936) stipule que le citoyen est obligé d'appartenir à une communauté reconnue par l'État et qu'il ne lui est pas permis « d'être régi en matière de statut personnel en dehors des lois de la communauté à laquelle il appartient » (Traboulsi, 1998 : 55). Les communautés ont donc reçu, par délégation de l'État, l'autorisation de dire le droit en matière de statut personnel (mariage, divorce, succession, filiation), ce qui explique que l'individucitoyen disparaît, aux yeux de l'État, derrière sa communauté d'origine.

5 Pour le mariage, l'incidence de cet état de fait est relativement simple: en l'absence d'une communauté de droit commun, il n'y a pas de mariage civil au Liban. En effet, seuls sont possibles les unions matrimoniales religieuses, donc entérinée par l'une des dix-huit communautés actuellement reconnues par l'Etat. La conséquence de cela est l'apparition depuis lors d'une pratique de « braconnage matrimonial » à l'étranger pour les couples qui souhaitent s'unir civilement, et notamment parmi eux les couples interreligieux. En effet, le système libanais qui confère à la communauté l'autorité sur le statut personnel de ses membres ne facilite pas les mariages intercommunautaires et rend les unions entre musulmans et chrétiens quasiment impossibles à moins d'une conversion. Chypre est ainsi devenue une destination idéale pour ces acteurs qui souhaitaient rompre avec l'ordre communautaire et représente en même temps une sorte de soupape pour un Etat qui trouve ainsi les moyens de conforter la version étroite du communautarisme, celui des seules communautés religieuses.

6 Cet État qui ne se dit pas laïc mais qui semble y aspirer n'a donc pas les moyens de protéger ses citoyens de leurs communautés. En tant qu'il est l'« expression d'un consensus entre communautés » (Assaf, $2000: 33$ ), il ne peut pas prendre parti pour un 
individu lorsque celui-ci affronte sa communauté, à plus forte raison si cette dernière contrôle son statut personnel. Cette relation problématique État-communautés prend corps juridiquement dans un décret-loi ( $n^{\circ} 112$ du 21.6.1959) qui impose à l'État de considérer l'équilibre confessionnel comme un impératif de tout premier ordre, une sorte d'intérêt supérieur de la nation qui prétérite, comme on l'a vu, l'égalité des droits politiques.

7 La genèse institutionnelle de ce rapport État-communautés est à rechercher dans les rapports de force que les élites marchandes beyrouthines avaient réussi à créer avec, puis contre la puissance mandataire. Au début des années quarante, l'entente entre leaders maronites et sunnites préfigura un partage du pouvoir. L'organisation confessionnelle des charges du pouvoir et de l'administration vint alors couronner et entériner l'hégémonie maronite fondatrice de l'État-nation libanais, notamment par la manipulation du recensement de 1932 (Maktabi, 2000). Cette hégémonie fut battue en brèche lors des accords de Taëf en 1989 au profit d'un système d'équilibre communautaire (Bahout, 1999) qui fait littéralement disparaître les individus au profit des acteurs-phares que sont les représentants des communautés. Cette quasisacralisation des communautés s'est lourdement fait ressentir depuis la fin de la guerre, le système politique se trouvant parfois pris en otage par les trois présidents, chacun incarnant sa communauté (Kassir, 2000).

8 La conséquence de ce nouveau rapport de forces, largement soutenu par la Syrie, est d'avoir conforté le communautarisme politique dans sa version la plus proche de l'ordre milicien (Picard, 1995), la pacification en plus. Ce double mouvement de renforcement communautaire et de distance entre communautés trouve une manifestation éclatante à propos du projet de "statut personnel civil unifié " proposé par le Président Hraoui en 1997. Ce projet tentait de concrétiser la mesure "provisoire» que l'article 95 de la Constitution stipulait à propos de la confessionnalisation des charges politiques. La dimension transitoire de cet article constitutionnel avait été reconduite tel quel en 1990, toujours dans l'hypothétique attente d'une "déconfessionnalisation ». Or, depuis les années cinquante, plusieurs tentatives de création d'une communauté de droit commun possédant un statut personnel de droit commun avaient échoué devant la résistance des hiérarchies religieuses musulmanes et chrétiennes. Le projet de E. Hraoui tentait à nouveau cette unification des statuts personnels au sein d'une communauté civile, donnant ainsi le choix aux citoyens de toute communauté d'intégrer la communauté civile. Ce faisant, l'État disputait le monopole des communautés religieuses, seules dépositaires de l'organisation du statut personnel des citoyens. En outre, le projet instaurait la possibilité du mariage civil ainsi que l'égalité entre hommes et femmes (Assaf, 1999).

9 L'échec de ce projet de statut personnel unifié et optionnel a laissé entrevoir non pas une opposition uniforme des élites communautaires avec la hiérarchie religieuse aux avant-postes, mais un affrontement entre communautés : au Conseil des ministres, les chiites, après concertation avec le président du Parlement, ont massivement voté en faveur du projet alors que les sunnites s'y sont opposés. Le premier ministre (sunnite) Rafic Hariri puis son successeur Selim Hoss ont tous deux contesté tant le projet que sa modalité d'adoption. Celui-ci avait été accepté par le Conseil des ministres à la majorité des deux tiers. Le premier ministre était alors chargé de le transmettre au Parlement, ce qu'il ne fit pas sous prétexte qu'un projet qui touchait à la coexistence entre communautés devait, selon la Constitution, faire l'objet d'un consensus au sein du 
Conseil des ministres et non d'un vote à la majorité des deux tiers. Or ce mode majoritaire avait été choisi parce qu'il n'était justement pas possible de trouver un consensus. Aussi faut-il noter que dans les prises de positions relatives à ce projet d'essence laïque, seuls les religieux sunnites «montèrent au créneau ». En revanche, les prélats chrétiens, d'abord perplexes, appuyèrent la position communautaire emmenée par le président de la République. Finalement, le blocage juridique provoqué par le Premier ministre eut raison du projet alors même que le Conseil des ministres avait entériné par 21 voix pour et 7 contre l'adoption du statut personnel unifié et optionnel (Najm, 2004).

10 Cet échec montre bien les limites de la laïcisation de l'ordre communautaire, et plus spécialement de la famille qui en constitue l'unité fondamentale. En outre, le débat avorté sur le statut personnel extra-communautaire ou civil illustre, outre la persistance du confessionnalisme, la nécessité de penser de manière non normatives les questions matrimoniales. Dans ce qui suit, nous nous proposons de poursuivre cette réflexion sur les unions matrimoniales à partir du cas des mariages libano-palestiniens. Ces derniers permettent en effet de réintroduire la problématique de l'identité collective (communautaire et nationale) à partir de trajectoires individuelles. Plus encore, ce type d'unions matrimoniales témoignent de processus spécifiques d'intégration au sein du tissu social libanais.

\section{Les unions matrimoniales libano-palestiniennes}

\section{A. Les conditions de l'enquête}

11 En raison de l'absence de données quantitatives ${ }^{2}$, mais également pour documenter les profils individuels, les discours et les perceptions des acteurs, nous avons procédé par entretiens semi-directifs auprès d'une soixantaine de couples libano-palestiniens. Rencontrés individuellement ou en couple, souvent en présence d'autres membres de la famille et très souvent à leur domicile, ces interlocuteurs se sont montrés extrêmement disponibles et, pour certains, disposés à évoquer des "problèmes » liés au mariage ou aux interactions avec l'autre groupe national, libanais ou palestinien.

Le corpus est composé d'une moitié environ de couples mariés dans l'après-guerre alors que les autres se sont unis durant les quatre décennies précédentes. Pour la plus grandes parties, ces mariages se sont effectués entre membres appartenant à la même religion (mais pas forcément à la même communauté religieuse) et seuls quatre d'entre-eux étaient des couples islamo-chrétiens. On peut relever d'une part que dans l'après-guerre civile, ces unions se font très largement entre musulmans (sunnite/ chiite/druze) et très marginalement entre chrétiens, le nombre de Palestiniens chrétiens au Liban étant relativement faible, de l'ordre de 8'000 individus environ. D'autre part, au niveau des 60 couples interrogés, force a été de noter le relatif panachage de genre rendant très ardu toute conclusion a priori sur le fait qu'il y aurait tendanciellement plus de femme palestiniennes qui épousent des hommes Libanais que d'hommes Palestiniens qui convoleraient avec des femmes Libanaises ${ }^{3}$. Etant entendu qu'une proportion significative des Palestiniens vivant actuellement au Liban habite hors des camps (43\%), notre recherche à tenté, dans un premier temps, d'équilibrer dans les mêmes proportions notre corpus de couples libano-palestiniens. Or, très rapidement, nous avons compris que ce phénomène était plutôt marginal au sein des 
camps et prenait une forme récurrente, celle de l'homme palestinien d'origine rurale et de la femme libanaise provenant d'une famille de la périphérie du camp et à faibles ressources économiques et culturelles. De surcroît, nos observations hors des camps nous ont montré la nécessité de tenir compte, au regard du contexte libanais, de la variété d'appartenance confessionnelle des conjoints libanais. Enfin, la diversification géographique de nos entretiens nous est apparue comme un des moyens de renforcer cette variance, puisqu'un mariage entre une chiite libanaise et un sunnite palestinien à Tyr n'a certainement pas la même valeur ou résonance que s'il a lieu aux abords de Chatila ${ }^{4}$. Aussi, nos entretiens ont eu lieu dans les environs des principaux camps (Sud, capitale, Nord, Bekaa), dans les principales villes côtières mais aussi dans le Metn où vivent plusieurs vieux couples libano-palestiniens chrétiens et plus marginalement au Nord et dans le Békaa.

Un autre préambule à cette enquête consiste à dire que si toutes les unions possèdent leurs dynamiques spécifiques, il est au moins un aspect que tous ces couples partagent: la nécessité du collectif familial qui impose son regard sur ladite union. Au-delà de l'aspect juridique des procédures au demeurant bien régulée pour les unions libanopalestiniennes ${ }^{5}$, c'est là une constante qui, comme nous le verrons plus bas, pose problème à certains couples vivant principalement dans le milieu urbain. En effet, la norme impose encore largement des schémas d'union majoritairement situés dans le réseau de connaissance, souvent au sein de la communauté et donc dans le groupe national. Principaux médiateurs de cette norme, la famille et, au sein de celle-ci, le père ou éventuellement le fils, frère du ou de la mariée et l'oncle maternel. Pourtant une relative liberté de choix existe et s'est étendue au réseau propre du jeune homme ou de la jeune fille. Face à l'irruption de cet étranger bien spécifique qu'est le Palestinien, les réactions des familles libanaises divergent comme nous le verrons en fonction de la nature des rapports réel ou idéel qu'ils entretiennent avec le groupe national palestinien. A l'inverse, bien plus rarement pour les familles palestiniennes un prétendant libanais pose-t-il problème en raison de son appartenance nationale. Mais généralement, le trait qui ressort de ces unions renvoie à une question d'acceptation et d'intégration, ici principalement analysée du point de vue du groupe national et du pays dans lequel vivent ces couples.

\section{B. Les Palestiniens au Liban : des relations tumultueuses}

Ce qui frappe l'esprit, dès que l'on engage une enquête de cet ordre, est l'épaisseur historique dont les relations sociales sont chargées. Aussi est-il bon de rappeler la genèse des relations actuelles entre Libanais et Palestiniens. Trois temps peuvent être dégagés: le premier s'étend jusqu'à la fin des années soixante, le second, qualifié d' "âge d'or » dure jusqu'en 1982 et le troisième se déploie à partir de cette époque jusqu'en 2005 en tous cas. On sait depuis plusieurs années que les relations entre individus et groupes ont été affectés par la transformation des "paysans en révolutionnaires" (Sayigh, 1979) qui a eu lieu avec l'amorce de la lutte armée antiisraélienne à partir du Liban, puis du fait de la montée en puissance de la Résistance palestinienne à la faveur de la défaite des armées arabes face à Israël en juin 1967. Parmi ces relations sociales, celle du mariage entre Libanais et Palestiniens a été relativement peu étudiée. Or, cette approche peut permet d'approfondir le regard sur les changements qu'il y a eu entre ces deux groupes nationaux et qui ont mené à la configuration d'après-guerre. 

matrimoniales entre Libanais et Palestiniens étaient assez largement circonscrites aux individus palestiniens - hommes ou femmes - chrétiens et/ou assez aisés. Ceci s'explique en grande partie par la politique à deux vitesses adoptée par les gouvernements libanais surtout durant les années cinquante. D'une part on parqua les Palestiniens les plus démunis dans des camps aux abords des villes: l'économie libanaise disposait ainsi de travailleurs journaliers bon marchés aux abords des villes. D'autre part, pour les Palestiniens plus aisés et mieux formés, souvent chrétiens, l'intégration s'opérait par l'obtention facilitée de la nationalité libanaise. Dans ce contexte, les unions matrimoniales répondaient à une exogamie sélective chez les Libanais et, pour les Palestiniens, notamment chrétiens, à une logique d'hypergamie ${ }^{6}$. Il faut alors se souvenir qu'en ce temps, le passeport Libanais devenait une valeur refuge et une source d'avantages. Les restrictions juridiques à l'égard des Palestiniens n'allaient du reste pas tarder à mettre ce fait encore davantage en exergue. palestinienne canalise les aspirations panarabes et que la puissance de la résistance palestinienne au Liban est consacrée par les Accords du Caire en 19697. Les stratégies matrimoniales semblent avoir également connu un tournant puisqu'on observe une nouvelle tendance à l'exogamie chez les Palestiniens. Leurs choix matrimoniaux s'orientent alors vers des secteurs de la société libanaise qui lui sont idéologiquement proches comme les formations de gauche favorable à la cause palestinienne et plus généralement les partis affiliés au Mouvement National emmené par le leader druze Kamal Joumblatt.

17 La plupart des couples interrogés, mariés durant cette seconde période, ont vécu leur union matrimoniale d'une manière très « intense ", au sens où l'époque commandait de «faire corps" avec le groupe et la cause palestinienne. Les lieux de rencontre témoignent de l'engagement politique qui préexistait souvent chez les deux conjoints et qui semblait créer les conditions d'une relation et d'une union.

18 Jusqu'au début des années quatre-vingt, les formes d'intégration des Palestiniens au Liban ont pu prendre des traits relativement variés mais toujours suivant une logique duale, propre à la divergence des options nationales qui se sont affrontées à partir du milieu des années soixante. Ainsi, les Palestiniens chrétiens étaient intégrés dans des réseaux essentiellement chrétiens (professions, lieux d'habitation, relations sociales) alors que les Palestiniens musulmans évoluaient à l'intérieur des réseaux marqué par leur engagement, participant souvent à des activités militantes plus ou moins encadrées par la Résistance palestinienne. Cette ligne de clivage a souvent recouvert une ligne confessionnelle avant de se manifester autour du projet de société lui-même : si en 1955 on se mariait avec un Palestinien parce qu'il était chrétien, en 1970, on épousait un Palestinien pour la cause que son identité nationale résumait.

19 L'invasion israélienne du Liban en 1982 brisa cette symbiose entre les deux groupes nationaux d'ailleurs déjà entamée par des divisions internes, notamment celle opposant Palestiniens et chiites du Sud-Liban (Khalidi, 1986). Le départ de la centrale palestinienne de Beyrouth puis du Liban annonçait le déclin des Palestiniens au Liban et ouvrit une dynamique de marginalisation brutalement entamée avec les rafles lancées par les services du président Gemayel, appuyé pour l'occasion par la milice des Forces Libanaises. En effet, des lois criminalisant les Palestiniens venus au Liban après 1948 ou rendant plus difficile d'accès le marché du travail furent ratifiées dès les 
premiers temps du mandat d'Amine Gemayel et les accords du Caire de 1969 abrogés en 1987.

\section{Vers l'institutionnalisation de la marginalisation: quelle intégration?}

Si les années quatre-vingt ont représenté un "creux» dans les relations libanopalestiniennes, on peut en dire autant des unions matrimoniales. Nous avons effectivement eu de la peine à trouver des couples mariés durant cette époque et, de l'avis de plusieurs interlocuteurs, ce type d'union était marginal du fait de la situation périlleuse que vivaient les Palestiniens du Liban durant ces années (affaiblissement politique et militaire, pauvreté accentuée, marginalisation sociale et harcèlement militaire avec la guerre des camps). Plus encore, les rares couples rencontrés, mariés durant ces années sombres, présentent un profil que l'on pourrait synthétiser de la manière suivante : il s'agissait d'individus déjà situés sur les frontières de leur groupe communautaire ou national tels des chrétiens maronites pro-palestiniens, des Palestiniens insérés dans le tissu social libanais ou d'autres individus atypiques de la haute bourgeoisie palestinienne et libanaise.

Dans l'après-guerre, on a pu relever un durcissement de la législation à l'égard des réfugiés palestiniens au Liban (al-Natour, 1993) Cela traduit un renforcement de la dynamique de marginalisation puisque les domaines touchés sont plus variés qu'auparavant : ils vont des déplacements aux emplois (la liste des métiers interdits n'a fait que s'allonger) en passant par l'accès à la propriété immobilière. L'incidence concrète de ces mesures sur les unions matrimoniales semble donc devoir être dissuasive, principalement lorsque l'homme est palestinien puisqu'il ne peut obtenir la nationalité libanaise. De surcroît, en suivant la représentation dominante qui veut que l'homme soit le pilier financier de la famille, marier un homme Palestinien annonce des difficultés supplémentaires pour la future famille puisqu'au Liban, il n'a pratiquement plus que les secteurs de l'agriculture et du bâtiment pour pouvoir gagner sa vie. Pourtant, de nombreux mariages ont lieu et montrent que tant les hommes que les femmes palestiniens pratiquent des unions avec des Libanais. Nous allons voir que cette contradiction entre statut et pratique renvoie à des logiques de durée, de catégories sociales et de choix personnels qui peuvent être en rupture avec la norme. En outre, ces types de mariages permettent d'observer, dans une catégorie urbaine d'acteurs dotés en diplômes et à revenus moyens, des réallocations de tâches au profit de la femme libanaise. Celle-ci doit assurer l'essentiel du revenu familial par son travail au moyen d'une inversion relative des rôles entre mari et femme. D'autres registres peuvent être convoqués pour subvenir aux besoins ordinaires. "Souvent, nous dit un informateur à Tyr, les familles ici reçoivent un mandat international chaque mois d'un fils ou d'un autre homme parti à l'étranger, en Suède par exemple ». Dans la Bekaa, nous avons vu que c'étaient des relations politiques ou des appuis (wasta) locaux qui liaient Libanais et Palestiniens. En d'autres termes, les difficultés auxquelles les couples libanopalestiniens font face ouvrent des possibilités d'intégration qui semblent dépendre de plusieurs facteurs (classe sociale, situation géographique, communauté, etc.) qu'il nous faut organiser à partir des types de couples que nous avons rencontrés.

Mes observations ont montré qu'il existe trois catégories relativement distinctes de couples libano-palestiniens, réparties en fonction de leurs ressources économiques et 
culturelles, chacune développant ses modalités propres d'intégration à l'univers environnant. La première est la plus fréquente. Elle concerne les couples vivant aux abords des camps palestiniens et dont les caractéristiques sociologiques sont une faible dotation en capitaux économiques et culturels ainsi qu'une forte interpénétration avec le lignage du conjoint palestinien. En outre, ce qui surprend l'observateur est probablement la «banalité» de ces unions libano-palestiniennes aux yeux des habitants de ces régions, notamment en raison de l'habitude que les uns ont de fréquenter et interagir avec les autres.

Le fait que ces unions soient relativement courantes s'explique d'abord par la proximité de lieu de vie des familles libanaises et palestiniennes qui facilitent les contacts entre partenaires potentiels. Or, dans ces univers où existe un certain respect des normes et des traditions notamment matrimoniales, les liens qui se développent avec le voisinage engendrent des unions matrimoniales. Il est donc courant pour les hommes d'aller faire une demande en mariage au père de la fille vivant dans la maison d'en face à moins que ce ne soit les parents du garçon qui fassent la démarche, comme l'explique Kamal ${ }^{8}$, un palestinien de la vieille ville de Saïda : «On s'est vu un jour... Puis une autre fois. Elle m'a plu et j'en ai parlé à mes parents qui ont fait la demande. Et on a fait un mariage traditionnel ». Sa femme explique: "Mes parents considèrent qu'il n'y a pas de différence entre Palestiniens et Libanais, c'est pour cela que notre mariage n'a pas posé de question. En plus, une cousine a épousé un de ses cousins ». Le fait qu'il y ait un échange (badal), parfois différé d'une génération, illustre une partie significative de ce premier type d'union. Ce qui par contre semble partagé par tous les couples dans ce milieu social, c'est la primauté du principe de proximité (voisinage) sur l'origine nationale.

Lorsqu'il y a difficulté ou contentieux, les raisons sont d'abord d'ordre "techniques »: second mariage de l'homme, fille encore à l'école au moment de la demande ou ressources du prétendant encore insuffisantes. Les oppositions parentales, lorsqu'elles relèvent de question d'appartenance, renvoient d'abord à l'appartenance confessionnelle. Enfin, très marginalement, l'appartenance palestinienne peut être la cause de l'opposition du père de la fille libanaise. Mais, au plus tard lors la naissance du premier enfant, les rapports beau-père/mari rentrent dans l'ordre. Ainsi, Rada, une chiite vivant à Tyr avec son mari explique: «mes parents se représentaient les Palestiniens comme des gens dangereux. Aussi, ils ont été surpris avec Salim car il est bien élevé. Pourtant, il a fallu que nous ayons un fils, notre premier enfant, pour que mon père l'embrasse vraiment $"$.

De manière générale, on peut donc noter une horizontalité dans les rapports sociaux, fondée sur une perception d'équivalence statutaire entre les deux groupes nationaux et, corrélativement, une faible emprise des discours médiatiques et politiques au sujet $\mathrm{du}$ " problème palestinien au Liban ».

Il faut dire que les difficultés que les uns et les autres connaissent (coupures d'électricité, chômage, mauvaise qualité de l'eau lorsqu'il y en a, mauvaises conditions sanitaires et couvertures médicales) renvoient à une précarité sociale et économique manifeste face à laquelle la solidarité prévaut. Pourtant, en matière d'alliance matrimoniale, il semble y avoir une limite, confessionnelle et communautaire, que les Palestiniens ne peuvent franchir sans soulever quelque opposition : le mariage avec des femmes chiites. Ahmad ${ }^{10}$ explique pourquoi sa mère chiite (libanaise) a essuyé plusieurs refus de ses parents pour pouvoir épouser Hasan, un palestinien sunnite: "Ce fut 
difficile pour la famille de ma mère d'accepter ce mariage. (...) C'est vraiment épouvantable pour une famille chiite de donner sa fille à un sunnite. Cela veut dire qu'elle va en enfer, elle et 40 de ses parents! ». Finalement la médiation d'un homme reconnu pour sa sagesse, religieux chiite de la notabilité locale, a permis d'obtenir, lors d'une rencontre au domicile du notable, le consentement des parents chiites en leur assurant que Hasan était « un homme d'excellente réputation ».

Cette forme d'intimité entre deux groupes différents parfois au plan communautairesur un sujet aussi sensible que le mariage ne peut s'obtenir que par l'effet du temps qui permet une sorte d' "appropriation" de l'Autre. Nous l'avons entendu comme par opposition dans la bouche d'une de nos interlocutrices, Roula, qui, à Tyr, expliquait en quoi les Palestiniens de Tyr sont mieux que les autres Palestiniens: "J'ai parfois rencontré des Palestiniens de Saïda ... mais ils sont différents ». En quoi ? «Bon, heu, si vous voulez, ils sont moins intégrés ou mélangés avec les Libanais, là-bas. Ils sont moins ouverts d'esprit ».

Ce signe nous a paru révélateur d'une qualité de relations entre Libanais et Palestiniens qui permet de parler d'intégration dans le tissu social environnant. Ce qui n'est pas peu dire au vu des antécédents guerriers et des contentieux de sang qui existent entre les uns et les autres, notamment entre ex-miliciens chiites et fedayins Palestiniens. Comme le résume Kifah, une ex-combattante palestinienne longtemps emprisonné par Israël et vivant à Beyrouth, « je me suis marié avec un chiite, hé oui, et pourtant les chiites, nous ne les avons pas beaucoup aimé dans la famille : un temps c'est vrai je les détestaient. Ils avaient tués mes frères et mon père quand même $»^{11}$. Certains interlocuteurs au Sud du pays ont cependant minimisé ces contentieux comme pour expliquer que, hors de la capitale, la guerre des camps ne les avait pas affectés. Cette volonté d'aplanir un passé parfois douloureux s'inscrit également dans ce processus intégratif. Un processus qui est lui-même situé dans un contexte d'exclusion sociale qui frappe tant les couples dont l'homme est Palestinien que ceux où il est Libanais. La précarité voire la pauvreté, pour cette catégorie d'acteurs à faibles capitaux culturels et économiques, renvoie à une série d'options de politique économique dans l'après-guerre dont il a été montré qu'elles renforçaient les processus d'exclusion sociales et économiques dans les segments de la population qui possèdent peu de ressources notamment économiques (Kochuyt, 2004 ; Ugland, 2003).

29 À l'opposé de l'espace social, là où revenus et dotations en capital culturels sont élevés et anciens de plusieurs générations, les unions matrimoniales libano-palestiniennes sont peu nombreuses et leurs auteurs sont des individus qui vivent des dilemmes identitaires saillants mais dont les incidences matérielles sont bien moindre que pour ceux de la catégorie précédente. Comme ils ne vivent en général pas au contact immédiat des Palestiniens, leur membre palestinien développe des sentiments complexes qui témoignent des difficultés à trouver une place qui leur permette d'être reconnu.

Ghada ${ }^{12}$, une franco-palestinienne élevée à l'étranger, est revenue au Liban vingt ans après en être partie. Fille d'un homme d'affaires très actif au sein de l'OLP, elle se retrouve mariée, peu de temps après son premier retour, avec un homme de la bourgeoisie marchande maronite. Elle devient alors très vite l'objet, comme elle le dit elle-même, «de petites vexations et de dépréciations parce que j'ai l'accent palestinien lorsque je parle l'arabe ». Le hiatus identitaire apparaît plus clairement à la faveur d'un événement commémoratif qui lui fait changer d'optique : 
31 «Je voulais vraiment m'insérer. Et puis un jour, il y a peu de temps, une amie m'a entraîné à une commémoration des massacres de Sabra et Chatila à l'UNESCO. J'ai découvert que mon accent n'avait plus aucun sens négatif, j'étais comme tout le monde, je me sentais à l'aise au milieu des Arabes, Palestiniens et Libanais. J'y ai aussi vu des chrétiens maronites qui ne ressemblent pas du tout à ceux que j'ai l'habitude de fréquenter. (...) Depuis, j'ai arrêté de corriger mes enfants lorsqu'ils prononcent un mot à la libanaise, je n'ai plus envie qu'ils vivent la même discrimination que moi. Il faut arrêter de traîner ce passé comme un fardeau ».

32 La conscience de son origine palestinienne comme "fardeau " la conduit donc à accepter, au vu du contexte libanais et du milieu maronite où sa famille vit, de ne plus contrôler le langage de ses enfants. D'autres exemples ont montré que certaines femmes palestiniennes, devenues libanaises par mariage, abandonnent complètement leur appartenance d'origine, comme s'il s'agissait, pour reprendre les termes qu'elles ont utilisés, d'«une autre vie», ou d'«un temps révolu» qui n'aurait "plus d'importance maintenant ». Mona ${ }^{13}$, palestinienne née au Liban dans les années 1950's dans un village proche de Saïda, mariée à un universitaire et vivant à Beyrouth semblait ainsi gênée (pour son interlocuteur surtout) de n'avoir, dans un premier temps rien à dire sur «cette époque» qui «ne (la) concerne plus». Puis, la ressouvenance aidant, elle «retrouva» son statut de palestinienne, détaillant longuement les discriminations dont sa famille avait été victime pour clore son propos par une affirmation claire : « même si j'ai obtenu la nationalité libanaise, mon sang est palestinien ».

33 Enfin, il faut noter que la bourgeoisie palestinienne qui, dans l'après-guerre civile, réalise des mariages avec des Libanais, est constituée d'hommes ou de femmes qui ont déjà la nationalité libanaise en raison de leur statut ou de leur confession, mais également en raison d'unions matrimoniales libano-palestiniennes antérieures au sein de leur famille. Ce second profil illustre une nouvelle forme d'exclusion par intégration : l'auto-exclusion volontaire et raisonnée du groupe palestinien. Toutefois, il faut en relever la dimension de genre, car c'est un phénomène qui ne touche que les femmes, les hommes ayant d'autres ressources, notamment financières, pour organiser par eux-mêmes une intégration au collectif libanais qui leur permet de conserver une vision positive et vivante de leur appartenance palestinienne. Le cas de Allan est à ce titre intéressant puisqu'il intègre un aspect récurrent lié à cette catégorie sociale-ci, celle des études et/ou de la vie à l'étranger avant un retour au pays. Né aux Etats-Unis de parents palestiniens aisés ayant vécu quelques années au Liban, il vient de se marier avec Mona, une femme libanaise qu'il a rencontré dans un groupe militant d'obédience socialiste lors de ses études. Après un premier voyage en Palestine, Allan décide de se mettre sérieusement à l'arabe et va ensuite donner des cours d'anglais dans une université palestinienne durant deux ans. Après son mariage avec Mona et du fait de la nationalité de cette dernière, ils sont obligés de vivre aux Etats-Unis. Mais ils décident d'un commun accord de "se rapprocher de la Palestine » en venant vivre au Liban. Ce faisant, Allan continue à re-construire son identité palestinienne en continuant les cours d'arabe et le travail militant pour la cause palestinienne.

34 Le troisième groupe significatif d'acteurs libano-palestiniens se trouve en milieu urbain et est constitué d'une figure récurrente, celle de l'homme palestinien ayant effectué des études et qui a rencontré sa future femme libanaise sur les bancs de l'université. En général, l'homme provient d'une famille vivant hors des camps et dont les ressources 
économiques ont été au moins suffisantes pour permettre aux garçons de la famille d'effectuer des études supérieures. Pour sa part, la femme libanaise est issue en général d'une famille peu dotée en capital culturel et ne vivant pas en milieu urbain. Elle a donc marqué une distance physique avec son milieu et affronte en général d'importantes résistances lorsqu'elle présente son futur époux à sa famille. Le cas de $\mathrm{Hana}^{14}$ et Naïm semble éloquent à ce titre.

Elle vient d'une famille chiite conservatrice de la Bekaa et lui d'un milieu bourgeois palestinien, universitaire mais très conservateur. Le mariage n'a pas été facile à faire accepter chez la famille libanaise car, explique-t-elle, « mes parents et même mes frères les plus progressistes n'envisageaient les Palestiniens que dans des camps, pauvres et sales » et, de surcroît, ses enfants allaient être Palestiniens. Du côté palestinien, la famille a d'abord refusé la perspective de cette union, car «ils voulaient quelqu'un qu'ils connaissent, qui soit du réseau des relations (...) au début, ils ne voulaient donc pas entendre parler de moi car en plus d'être libanaise, je suis chiite ». Il lui a fallu environ 4 ans pour faire accepter l'idée de leur union mais, contrairement aux couples à faibles capitaux culturels et économiques, le fait que la fille soit chiite n'a apparemment pas été un sujet de la discorde au sein sa famille. La grande question qui semble préoccuper Hana est celle de l'avenir de son jeune enfant: stratégie scolaire, séjour chez une grand-mère aux Etats-Unis, toutes les idées sont bonnes pour contourner le handicap de l'identité palestinienne.

Ce souci de l'avenir de l'enfant est très répandu chez ces acteurs universitaires et encore peu assurés de revenus confortables. Et l'opposition du beau-père au mariage provient en général de la difficulté que les parents de la femme libanaise ont à s'imaginer avoir des petits-enfants palestiniens. Ainsi, Yahyah ${ }^{15}$, raconte les cinq années de tractations avec son beau-père qui ont failli mal se finir :

«Ma femme lui a dit souvent qu'elle voulait se marier avec moi et moi j'insistais et je revenais à la charge. Rien ne changeait dans sa tête. Un jour, on a fait un projet d'enlèvement avec ma femme. Lui a entendu parlé de cela et m'a téléphoné en me disant qu'il allait me tuer si je ne quittais pas le territoire du Liban avant le lendemain. Le soir même, je suis allé chez lui pour lui dire qu'il choisisse lui-même : soit il me tuait, soit il me donnait sa fille en mariage. Et là, il m'a embrassé et m'a dit d'accord ».

Parfois, l'exclusion familiale s'installe dans la durée et rend la vie au Liban de moins en moins attrayante. Le cas de Jamal ${ }^{16}$, assistante en salle de chirurgie dans un hôpital privé de la capitale, est typique de ce processus de rupture qui va en s'approfondissant. Elle est libanaise, de confession du druze - ses parents vivent dans un village du Chouf et elle a décidé de se marier, sans leur consentement, avec un palestinien travaillant à l'Université américaine. La norme de la montagne druze, qui proscrit les unions matrimoniales hors du cercle de la communauté, lui est devenue tellement étrangère qu'elle n'a pas hésité à changer de communauté et à devenir sunnite pour pouvoir se marier, le mariage civil n'ayant pas été possible pour des raisons administratives. Coupée de sa communauté et surtout de sa famille qui n'est pas venu à la célébration du mariage, elle paraît maintenant prête à se couper de son pays pour assurer l'avenir de son mari et de ses enfants: "Je suis prête à vivre à l'étranger car il faut n'importe quelle autre nationalité pour eux, pour qu'ils puissent vivre normalement ».

On voit donc que l'intégration sociale des Palestiniens par alliance matrimoniale avec des citoyens libanais ne résout de loin pas, pour ces jeunes couples d'universitaires, le problème fondamental qui reste, comme pour la première catégorie examinée, l'accès 
au marché du travail. La raison principale ici est le fait que, dans ces unions, l'homme est palestinien; il ne peut donc pas tirer profit professionnellement de son union avec une libanaise ${ }^{17}$ puisqu'il ne reçoit pas la nationalité libanaise par son mariage, celle-ci n'étant transmise que par l'homme. Ce troisième type d'union qu'on observe dans l'après-guerre est un phénomène urbain, lié à la capitale, et qui engendre très souvent une rupture assez importante avec les familles, cela au risque de conduire à un isolement social. Enfin, la nationalité palestinienne y est perçue comme un handicap qu'il faut contourner, notamment pour que les enfants ne se retrouvent pas avec un horizon professionnel très fermé. Une de nos interlocutrices libanaises a ainsi trouvé comme solution de partir en voyage en Grande-Bretagne pour y accoucher de ses deux enfants, ce qui leur a permis d'acquérir la nationalité britannique puisque ce pays applique le droit du sol (jus soli). Le souci de l'intégration professionnelle que les membres de ce troisième groupe d'acteurs libano-palestiniens manifeste leur fait choisir des options assez radicales (marginalisation familiale, déplacement à l'étranger, exil). Celles-ci, s'apparentent, pour les conjoints palestiniens, à des formes de négations ou d'auto-exclusion du groupe national palestinien.

\section{Conclusion}

40 Notre hypothèse de départ posait que le mariage entre Libanais et Palestiniens fonctionne, dans l'après-guerre, comme un système de pratiques intégratif mais qui, dans le même temps reconduit des formes d'exclusion déjà observables dans la sphère économique et au travers de lois restrictives à l'égard des Palestiniens. Pour défendre cette idée, nous avons, dans un premier temps, posé le cadre des relations sociales et matrimoniales au Liban en montrant la prégnance du régime communautaire sur l'ordre social et politique. Ensuite, nous avons présenté les diverses temporalités de la question palestinienne au Liban, en partie dépendante de cette logique communautaire, puis situé trois catégories d'acteurs libano-palestiniens de l'après-guerre: les plus démunis, le plus aisés et un groupe hybride ayant peu de ressources financières mais possédant des diplômes.

41 Nous avons pu voir que les formes d'exclusion consécutive à l'intégration sociale via le mariage avec un conjoint libanais sont de deux ordres, selon les segments sociaux où ces unions matrimoniales se déroulent. Pour le premier profil, assez largement représentatif de ce phénomène dans l'après-guerre, c'est la localisation du mariage au sein de groupes économiquement et socialement précarisés qui reconduit l'exclusion sociale et économique des acteurs. Dans ce contexte, l'identité palestinienne intervient peu comme variable déterminante de cette exclusion, sauf dans les cas spécifiques des couples libano-palestiniens des camps.

Le second profil laisse apparaître un trait commun au-delà de la différence en ressources économiques qui caractérisent ses acteurs : tous se livrent, à des degrés divers, à des formes de négations de leur appartenance palestinienne, et s'autoexcluent de leur identité nationale d'origine. Il faut toutefois faire une distinction au sein de ce groupe pour voir que les plus aisés et notamment parmi eux les hommes possèdent des moyens financiers pour éviter ce processus de négation de leur identité palestinienne que le processus intégratif entraîne lorsqu'il s'agit des femmes. A contrario, les moins aisés de ce second profil se trouvent à la conjonction de deux modalités d'exclusion que l'on a pu observer : ces couples libano-palestiniens, éduqués 
mais peu dotés en ressources financières, s'auto-excluent du groupe palestinien (et parfois même du groupe libanais) pour pouvoir assurer un meilleur avenir à leur famille et leurs enfants. Mais, dans le même temps, pour ceux dont le mari est palestinien, ils sont exclus du collectif libanais puisque les hommes palestiniens, comme on l'a vu, se trouvent nettement marginalisés sur le marché du travail libanais.

$\mathrm{Au}$ terme de ce travail, nous serions tenté de dire que tout se passe comme si les pratiques sociales venaient contredire une législation qui, en discriminant les Palestiniens, érige une barrière entre Libanais et Palestiniens et pénalise celles et ceux qui veulent contracter des mariages "mixtes». Le phénomène des unions libanopalestiniens montre que l'interpénétration de ces deux groupes nationaux a pris de vitesse tant les lois que les discours politique: dans la catégorie sociale la plus nombreuse à pratiquer ce type de mariage, parmi ceux qui vivent avec les Palestiniens, il s'est déjà normalisé.

\section{BIBLIOGRAPHIE}

Assaf G., 1999, « Système communautariste et déconfessionnalisation : problématique de la mutation du système politique libanais ", Travaux et Jours, $\mathrm{n}^{\circ}$ 64, pp.43-73.

Assaf G., 2000, « Les bases constitutionnelles de la liberté de religion dans un pays multicommunautaire : le cas du Liban ». In Les droits de l'homme au seuil du troisième millénaire. Mélange en hommage à Pierre Lambert, Bruxelles, Bruylant, pp.19-35.

Bahout J., 1999, « Du pacte de 1943 à l'accord de Taëf », in Hannoyer J. (dir.), Guerres civiles, ParisBeyrouth, Khartala-Cermoc, pp.299-313.

Breteau C.H., Zagnoli N., 1993, « Le sang et la terre », in Breteau C.H., Zagnoli N. (dir.), Production, Pouvoir et Parenté dans le Monde Méditerranéen, Paris, Geuthner, pp.11-77.

Cohen J., 1999, « Intégration : théories, politiques et logiques d'Etat », in Dewitte P. (dir.), Immigration et intégration : l'état des savoirs, Paris, PUF, pp.32-42.

Kassir S., 2000, « Dix ans après, comment ne pas réconcilier une société divisée ? ", MaghrebMachrek, $\mathrm{n}^{\circ} 169, \mathrm{pp} .6-22$.

Khalidi R., 1986, Under Siege: PLO Decisionmaking During the 1982 War, New York, Columbia University Press, 241 p.

Kochuyt T., 2004, « La misère au Liban : une population appauvrie, peu d'Etat et plusieurs solidarités souterraines », Revue Tiers Monde, Tome XLV, n 179, pp.515-539.

Maktabi R., 2000, "State Formation and Citizenship in Lebanon », in Butenchon N.A., Davis U., Hassassian M. (dir.), Citizenship and the State in the Middle East, Syracuse New York, Syracuse University Press, pp.146-178.

Meier D., 2005, « Contribution à une problématique de l'identité sociale. Le cas des couples libano-palestiniens au Liban », Maghreb Machrek, n 184, pp.105-122. 
Najm M.-C., 2004, « Pour une législation civile unifiée de la famille au Liban », Travaux et Jours, $\mathrm{n}^{\circ}$ 74, pp.131-172.

Natour al- S., 1993, Les Palestiniens du Liban, Beyrouth, Dar al-Taqqadom al-arabi, 229 p.

Picard E., 1995, «Les habits neufs du communautarisme libanais », Cultures \& Conflits, n¹5/16, pp. 49-70.

Picaudou N., 1989, La déchirure libanaise, Bruxelles, Complexe, 274 p.

Sayigh R., 1979, Palestiniancs : From Peasants to Revolutionaries, London, Zed Press, 256 p.

Traboulsi I.A., 1998, « De Nicosie à Beyrouth: le mariage civil au Liban », Travaux et Jours, nº 61, pp.53-63.

Ugland O.F. (dir.), 2003, Difficult Past, Uncertain Future. Living Conditions Among Palestinian Refugees in Camps and Gatherings in Lebanon, Oslo, FAFO, n 409, http://www.fafo.no/pub/rapp/409/409.pdf, 8 mai 2009.

\section{NOTES}

1. Nous prenons appui sur la tradition de l'école de Chicago, qui conçoit l'intégration/ assimilation à partir de multiples dimensions, notamment celles des mariages et du tissu socioéconomique (Cohen, 1999).

2. Selon notre estimation, basée sur des sources officieuses obtenues à l'UNRWA (Beyrouth), il y aurait eu chaque année entre 400 et 500 mariages libano-palestiniens durant les années 1990. Par ailleurs, un tiers des réfugiés palestiniens de la troisième génération aurait un parent Libanais (Meier, 2005).

3. Cet aspect genre n'est évidemment pas anodin au Liban puisque la femme libanaise, en raison de l'ordre social et juridique patrilinéaire, ne peut aucunement transmettre sa nationalité à son conjoint, ni même à ses enfants si le père est d'une autre nationalité. Un débat important à ce sujet à été soulevé de longue date par des associations de défense des droits des femmes mais les réticences socio-politiques semblent freiner toute concrétisation politique pourtant souhaitée par plusieurs ministres actuels, dont le ministre de l'Intérieur Ziyad Baroud.

4. En effet, le camp de Chatila a subi les assauts des miliciens chiites de Amal durant la guerre des camps (1985-1988) ce qui rend problématique la présence de chiites dans ce camp. Pourtant une mutation s'opèrent depuis quelques années : la présence du Hezbollah à Chatila lors de la commémoration en 2003 des massacres des Sabra et Chatila (1982) en témoigne.

5. Sur cet aspect et pour les détails des profils sociologiques des couples libano-palestiniens, on se reportera à notre article (Meier, 2005).

6. Un groupe social pratique l'hypergamie lorsqu'il s'estime dominant. En effet, au lieu d'échanger/donner des femmes, il se contentera d'en prendre dans un autre groupe jugé inférieur. Cette stratégie matrimoniale correspond assez bien à la pratique des chrétiens libanais dans les relations libano-palestiniennes des années 1950 (Breteau \& Zagnolli, 1993).

7. Ces Accords octroyaient aux réfugiés palestiniens outre un accès ouvert au marché du travail libanais le contrôle exclusif sur les camps, la reconnaissance de l'implantation militaire au sud et le droit de mener des opérations anti-israéliennes à partir du territoire libanais en concertation avec l'armée libanaise (Picaudou, 1989).

8. Entretien effectué à Saïda en septembre 2002

9. Entretien effectué à Beyrouth en septembre 2001

10. Entretien effectué à Tyr en janvier 2004.

11. Entretien effectué à Khaldé en septembre 2002. 
12. Entretien effectué à Beyrouth en septembre 2001.

13. Entretien effectué à Beyrouth en septembre 2001.

14. Entretien effectué à Beyrouth en septembre 2001.

15. Entretien effectué à Beyrouth en novembre 2003.

16. Entretien effectué à Beyrouth en septembre 2002.

17. Nous avons cependant rencontré quelques hommes qui utilisent les réseaux familiaux et professionnels de leur femme pour développer des activités commerciales, notamment dans la vente.

\section{RÉSUMÉS}

Dans cet article, l'auteur tente de démontrer que, dans le Liban d'après-guerre, les unions matrimoniales entre Libanais et Palestiniens fonctionnent comme un système de pratiques intégratif tout en reconduisant les formes d'exclusion déjà à l'œuvre dans les sphères économique et juridique. Pour développer cette hypothèse, il commence par montrer la place et les enjeux du mariage dans la société libanaise en regard du poids social et politique des communautés. Dans un second temps, il examine les alliances matrimoniales libanopalestiniennes à partir d'un corpus d'entretiens permettant l'appréhension de la problématique dans une perspective diachronique. Ces pratiques matrimoniales mettent au jour différents types de groupes libano-palestiniens dans l'après-guerre ainsi que, pour la majorité d'entre-eux, les logiques d'exclusion qui caractérisent leur intégration sociale.

In this paper, the author tries to sustain the hypothesis that in the Lebanon post-civil war, mixed marriages between Lebanese and Palestinians work like a practical system that integrate social actors but in the meantime reshuffle exclusion items that are still working in economic and law fields. To highlight this idea, he first shows the place and the stakes of marriage in the Lebanese society regarding the weight in social matters and in politics of these communities. Then, secondly, he examines Lebanese-Palestinian matrimonial ties through a set of interviews which allow one to understand the case within a historical background. The matrimonial unions tend to show different kinds of groups of mixed couples in the post-civil war. They also show that the majority of the Lebanese-Palestinian couples are experiencing social exclusion as a core process of their integration.

Der Autor dieses Artikels versucht darzulegen, dass die im nachkrieglichen Libanon beschlossenen Ehen zwischen Libanesen und Palästinensern wie ein integratives System funktionieren und alle Arten von bereits vorhandenem Ausschluss, sei es ökonomisch oder juristisch beibehaltet. Zum Ausbau dieser Voraussetzung zeigt er zuerst den Platz und den Einsatz der Ehen in der libanesischen Gesellschaft, mit Betracht auf das soziale und politische Gewicht beider Gemeinschaften. Im zweiten Teil untersucht er die libano-palestinensischen Ehebündnisse aufgrund verschiedener Gespräche, welche das Problem in ein diachronisches Licht werfen. Diese Heiratsgewohnheiten bringen die verschiedenen Arten von nachkrieglichen libano-palestinensischen Gruppen an den Tag und, für die meisten, die Logik der Aussetzung, welche die soziale Integration kenntlich macht. 
INDEX

Schlüsselwörter : Ausschluss, Ehen, Integration, Libanon, Nähe, Palästinenser

Keywords : exclusion, integration, Lebanon, marriages, Palestinians, proximity

Mots-clés : exclusion, intégration, Liban, mariages, Palestiniens, proximité

\section{AUTEUR}

DANIEL MEIER

Institut de Hautes Etudes Internationales et du Développement, Genève -

daniel.meier@graduateinstitute.ch 